\title{
Clever chip designs for diagnostics
}

Steady innovation in device design for diagnostic assays provides substantial performance gains.

A few decades ago, microfluidics was portended to lead a revolution in molecular analysis. Simple fluid kinematics, fast reaction times, the ease of prototyping and fabricating the devices (for example, via injection moulding or embossing), and the use of cheap and easy-to-handle materials (in particular, polydimethylsiloxane) was expected to unleash game-changing applications. Notwithstanding innovations such as organs-on-chips, paper microfluidics and open microfluidics, the reality is more modest: microfluidic chips remain of high academic interest, yet except for simple immunoassays, blood chemistry and next-generation sequencing, they are not commonplace in industry or in the clinic. This stems in part from engineering challenges towards the cheap mass production of fluidic microchips and from difficulties in finding new 'killer' applications for them. Yet the small volumes of samples and reagents needed, the precise control of flows, and the possibilities for assay automation and integration, make fluidic microchip technology particularly promising for molecular diagnostics, especially at the point-of-care. For killer uses to drive technological development forward, substantial gains in assay performance (in particular, detection sensitivity, specificity, concentration range and speed), and in robustness and operation with respect to available assays, have to be demonstrated. Sometimes this requires the clever combination of currently available technologies, or only relatively simple tweaks in chip design, to eliminate performance bottlenecks or enable new biomedically relevant readouts.

Three papers included in this issue exemplify this point. In one of them, Kiana Aran and co-workers describe a new microdevice and assay for nucleic-acid testing that does away with the need for amplification. Nucleic-acid testing for pointof-care applications is typically performed by amplification-based methods that rely on the quantitative polymerase chain reaction and require complex sample preparation, costly reagents or complex signal detection. Aran and colleagues combined the sensitivity and ease of operation of a graphene-based field-effect transistor with the specificity of CRISPR by immobilizing, on the graphene layer, deactivated Cas9

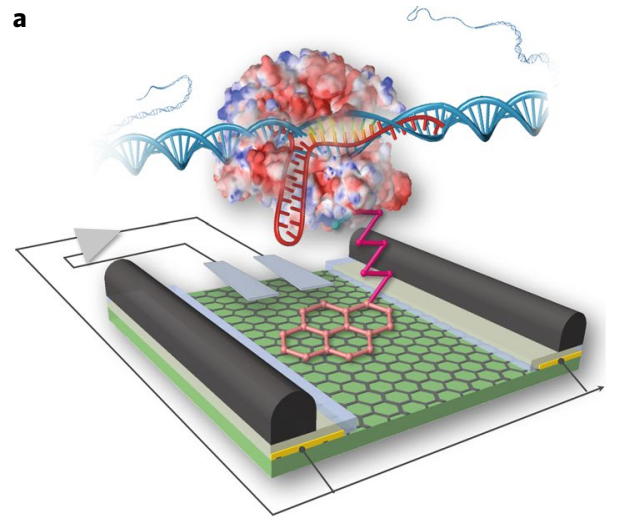

b
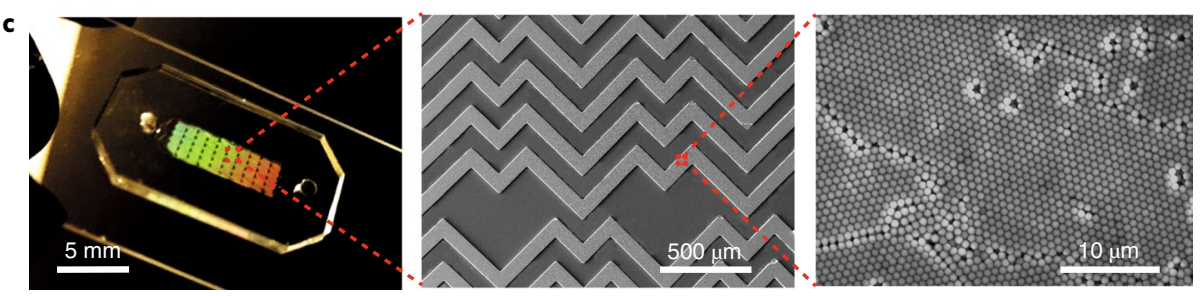

Fig. 1 | Chip designs for better diagnostics. a, A graphene-based field-effect transistor for the electrical detection of nucleic acids without the need for amplification. b, A microfluidic chip with porous herringbone-patterned surfaces for the sensitive detection of tumour extracellular vesicles. c, A microfluidic chip with Y-shaped channels for the assessment of the metastatic propensity of cancer cells. Figure reproduced from Hajian, R. et al. (a), Yankaskas, C. L. et al. (b) and Zhang, P. et al. (c), Springer Nature Ltd.

paired with a target-specific guide RNA (Fig. 1a). The sensitivity of the conductivity of graphene to biomolecular recognition on its surface endows the chip with sufficient sensitivity to detect the selective hybridization of target DNA with the immobilized Cas9 complex in intact yet purified genomic samples from a buccal swab in just 15 minutes. The researchers also show that the chip can detect two distinct mutations at exons commonly deleted in individuals with Duchenne muscular dystrophy. Importantly, because of the specificity of the Cas 9 complex (determined by the guide RNA), the chip can be 'programmed' to detect most DNA sequences.

The two other papers report chip designs that enable better diagnostic performance in the detection or phenotyping of cancer cells in patient samples. Yong Zeng and colleagues describe a microfluidic chip for the detection of extracellular vesicles (via antibodies for proteins on their surface) secreted by tumour cells in plasma samples from patients with early-stage ovarian cancer. The key aspect of the chip's design that makes it so sensitive (the device can detect 10 vesicles per microlitre of sample, a concentration unachievable with standard microfluidic chips) is a particular architecture: nanoporous surfaces, made of silica nanoparticles, arranged in a herringbone pattern (Fig. 1b). This architecture, fabricated by means of guided colloidal self-assembly, promotes flow patterns that enhance the interaction between the vesicles and the (antibodyconjugated) surfaces, increases the surface area available for vesicle binding, and reduces the resistance to flow (the interstices between nanopores drain the boundary layer).

Simpler microfluidic-chip designs can also lead to diagnostic advantages, such as the determination of the metastatic propensity of a primary tumour, which cannot be reliably done via standard 
pathology. Konstantinos Konstantopoulos and colleagues report a chip for the assessment of the metastatic potential of breast tumour cells derived from patient biopsies and expanded into tumour xenografts in mice. The researchers designed the microfluidic chip with Y-shaped channels of differing diameter to mimic a basic aspect of the stromal extracellular matrix through which metastatic tumour cells must migrate: the heterogeneity of cross-sectional areas of the pores in the matrix. The cells were fed into a $20-\mu \mathrm{m}$-wide channel, and those migrating to the bifurcated ' $\mathrm{Y}$ ' side of the channel could do it either through a $10-\mu \mathrm{m}$-wide channel or a narrower tube $3 \mu \mathrm{m}$ in diameter (Fig. 1c). An optimal assay duration (12-14 hours) and threshold percentage of migratory cells (7-9\%; migratory cells were defined as those that reached the bifurcated channels) led to $96 \%$ accuracy for the classification of the metastatic potential (low or high) of the breast cancer cells.

Steady innovations in the design of channels, valves, pumps, sensors and microelectronics, in microchips and in the materials and manufacturing processes for them, will increasingly make them better for diagnostic applications. However, in academic labs device robustness and reliability are less of a priority than flexibility in device design, fabrication and operation. The chips highlighted in this issue are thus only proof-of-concept evidence that rational chip design can enable better diagnostic assays. In the search for a killer commercial application, in addition to performance parameters such as sensitivity, specificity and speed, a great deal of development work would need to address factors such as consistency of performance, cost, ease-ofuse, throughput, and device integration with specific industrial or clinical processes. A clever chip might give it a head start.

Published online: 7 June 2019

https://doi.org/10.1038/s41551-019-0418-Z 